\title{
Effect of slab width on choice of appropriate casting speed in steel production
}

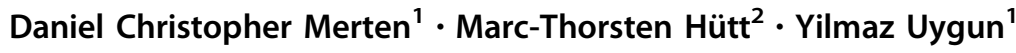

Received: 19 July 2021 / Revised: 20 September 2021 / Accepted: 24 September 2021 / Published online: 21 January 2022

(C) The Author(s) 2022

\begin{abstract}
In steel continuous casting (CC), the choice of the appropriate speed at which the slab is cast can be influenced by many different factors and phenomena. While the slab thickness seems to have the biggest impact, other features like the slab width have been consistently overlooked. In fact, the slab width practically limits the casting speed via the mass flow constraint which governs the input and output balance at the tundish. Here, we present a case study that aims at analyzing steel production data from the perspective of casting speed constraints. By studying the speed fluctuations of an industrial $\mathrm{CC}$ machine, we identify a strategic regime change toward a stricter consideration of the mass flow constraint. The regime change manifests itself in a significant increase in the correlation between the actual casting speed and the maximal speed associated with the mass flow constraint. On the surface, taking greater account of the input and output balance at the tundish has reduced the productivity of the continuous caster; however, one can argue that the lessened yield is compensated by a diminished risk of eventual slab breaking. From the perspective of this trade-off, we establish a visualization technique that enables us to pinpoint the boundary beyond which one strategic regime becomes economically more advantageous than the other.
\end{abstract}

Keywords Continuous casting · Casting speed $\cdot$ Slab width $\cdot$ Slab thickness $\cdot$ Mass flow constraint $\cdot$ Machine length constraint

\section{Introduction}

Among all metallic materials, steel is the most prominent with respect to commercial applications by far. Since the industry-wide adoption of continuous casting (CC) in the 1960s [1], continuous casting has emerged as the most commonly used steel production technique [2]. Over the years, the $\mathrm{CC}$ process has frequently been updated in the search for faster casting speeds and, therefore, ultimately greater economic returns [3]. Nowadays, most continuous casters work at speeds between 1.0 and $2.5 \mathrm{~m} / \mathrm{min}$ [3]. However, casting speeds can go up to $8 \mathrm{~m} / \mathrm{min}$ in some cases [1]. Primarily, the maximum possible speed is limited by the length of the caster, because the nascent steel slab

Daniel Christopher Merten

d.merten@jacobs-university.de

1 Department of Mathematics and Logistics, Jacobs University Bremen, Bremen 28759, Germany

2 Department of Life Sciences and Chemistry, Jacobs University Bremen, Bremen 28759, Germany has to be solidified sufficiently prior to leaving the machine, or else the slab shell will not be thick enough to hold the liquid steel inside [4]. Having said that, it is not entirely clear to what extent that other planning-relevant variables (e.g., slab width) are taken into account by the casting speed calculations in real-life factory environments.

Steel plant managers are regularly confronted with a trade-off decision, where higher casting speeds signify better productivity but come with an increased risk of breakouts [5]. On top of that, they should ideally keep the casting speed constant, as fluctuating casting speeds compromise the process quality $[6,7]$. Nevertheless, ensuring stable casting speeds is very difficult in practice, since the choice of the appropriate casting speed may not only be influenced by the slab thickness/machine length constraint, but could also be affected by the slab width, as we will demonstrate throughout this article. More precisely, the slab width enters the equation via the so-called mass flow constraint (see Fig. 1), which implies that the mass flow of liquid steel supplied through the ladle must not be outrun by the rate at which the continuous caster retracts steel 

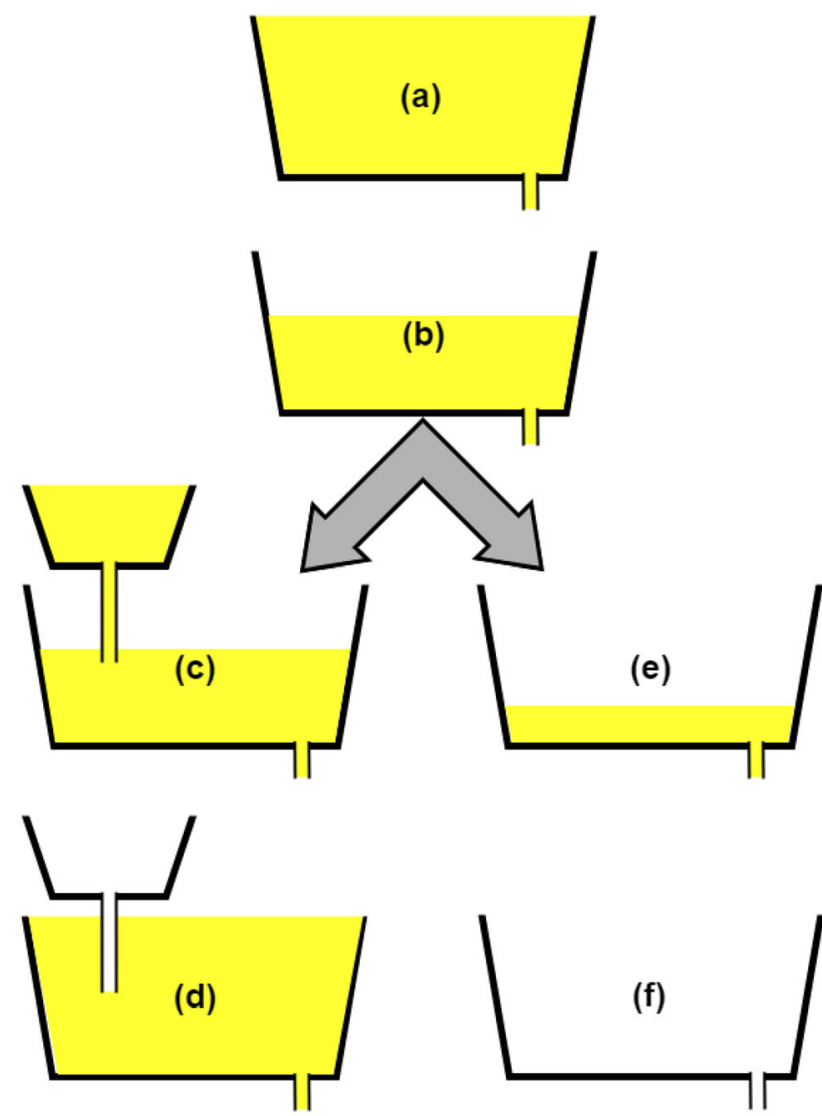

Fig. 1 Visualization of mass flow constraint

from the tundish or else the solidifying slabs are starting to break.

In Fig. 1a, a full tundish is shown. From this tundish, liquid steel flows through the mold and is eventually solidified. As the steel is retracted, the tundish slowly gets emptier (Fig. 1b). If new steel is provided through a ladle, the casting speed can be maintained (Fig. 1c, d). If no new steel is supplied, the casting speed needs to be slowed down (Fig. 1e), or otherwise, the tundish runs dry (Fig. 1f), and the production process may come to an abrupt halt due to slab breaking. This constraint has been consistently overlooked in Sect. 2, although it appears to be of high practical importance.

Our analysis is built on industrial data from a recently commissioned steel factory that experienced modifications regarding the mass flow constraint after having undergone a build-up production phase. The data have been anonymized according to a non-disclosure agreement with the steel manufacturer. Based on historical production records from a CC machine, casting speed fluctuations are analyzed in the context of this constraint. Furthermore, we introduce a visualization method that allows us to reverse-engineer the strategic decision resulting in a stricter consideration of the mass flow constraint and, thereby, the slab width. In this sense, the mass flow constraint as well as the aforementioned trade-off between productivity and quality concerns that have triggered this strategic decision will mainly be assessed by performing the following two steps: (1) identifying disparate production regimes that differ in the degree to which they respect the mass flow constraint and (2) measuring the mean production output of these regimes under the assumption that violations of the mass flow constraint cause slab break. Contrasting the output of the continuous caster before and after the strategic regime change will reveal the mass flow threshold beyond which steel plant managers suspect serious slab quality issues and an output decline.

\section{Related work}

Here, we provide an overview of the state of research at the intersection of slab-dependent casting speed limitations and casting speed-related quality concerns. While it remains rather unexplored in what way planning-relevant parameters [8, 9] like the slab carbon content [10] or the slab width determine the casting speed, and a substantial amount of literature links the casting speed to the slab thickness. Several papers describe how increasing casting speeds leads to thinner slab shells [11-13] which, by numerous authors, is attributed to the fact that at high casting speeds, the slabs have less time to solidify and exhibit a longer metallurgical length [14-16]. Some attempts at mathematically formalizing the relationship between the slab thickness and the casting speed are presented, for example, by Thomas [17] and MiłkowskaPiszczek et al. [18].

Apart from shell thinning and potential subsequent shell rupture, Li and Thomas [5] have discussed an extensive list of quality issues that restrict the casting speed including bulging, sticker breakouts, and cracks. In addition, various studies have investigated the effect of the casting speed on a number of different slab defects such as hook structures, particle inclusions, and oscillation marks. Whereas problems like bulging and breakouts seem to intensify at higher casting speeds [19, 20], Li et al. [20] and Lee et al. [21] reported a reduction in both the hook depth and the inclusion index/number of entrapped particles with growing casting speeds for speeds below 1.8 and $2.5 \mathrm{~m} / \mathrm{min}$, respectively. Their results are further backed up using an anti-correlation of the casting speed and the entrapment probability at the submerged entry nozzle revealed by $\mathrm{Wu}$ et al. [22]. Similarly, the depth of oscillation marks is found to decrease as the casting speed increases [21, 23].

As opposed to these thickness- and quality-associated casting speed limitations, there are hardly any articles that connect the slab width with the casting speed. Fu et al. [24] 
illustrate the impact of the casting speed on slab broadening, whereas Dauby [25] and Deng et al. [26] explain how the ratio of casting speed and mold width guide the steel flow pattern. However, no single piece of literature discloses to what degree the slab width dictates the maximum possible casting speed (e.g., via the mass flow constraint).

\section{Methods}

As evident through Sects. 1 and 2, our principal goal is to determine how the mass flow constraint and, thereby, the slab width affect the casting speed. By extracting the relevant features from a large industrial data set, we show that an analysis of the trade-off between productivity and slab breaking concerns has led to a more stringent consideration of the mass flow constraint within the factory of interest. Below, the characteristics of the industrial data are addressed briefly, and we will outline our methodological approach in detail.

Step 1 Computation of casting speeds and comparison with randomized casting speeds

The production data comprise values for the width, thickness, weight, and casting duration of over 100,000 slabs which are labeled with identification numbers corresponding to casting heats and sequences (see Table 1). From these values, the actual casting speed at which each slab is planned to be processed could be calculated, as well as two theoretically maximum possible casting speeds based on both afore-mentioned casting constraints, namely the machine length constraint $\left(v_{\text {solid }}\right)$ as well as the mass flow constraint $\left(v_{\text {mass }}\right)[17,18]$.

$v_{\text {solid }}=\frac{k \times l_{\max }}{\theta^{2}}$

$v_{\text {mass }}=\frac{m}{d \times w \times \theta \times \rho}$,

where $k$ is the machine-specific cooling capabilities; $l_{\max }$ is the length of the continuous caster; $\theta$ is the slab thickness; $m$ is the slab mass; $d$ is the slab casting duration; $w$ is the slab width; and $\rho$ is the steel mass density.
Afterward, we examine the distribution of the actual casting speeds visually and compare the actual intra-sequence speed fluctuations to a hypothetical situation (or null model) where the casting speeds are randomly assigned to sequences through repeated shuffling of the sequence identification labels. With this numerical experiment, we can understand whether the casting machine operators make an effort to keep the casting speed stable within the production sequences. If the operators appear to regulate the casting speed, we can proceed to assess the reasons (e.g., machine length, mass flow constraint) behind speed fluctuations.

Step 2 Correlation analysis and identification of production regimes

Now, in order to facilitate the evaluation of correlations between the actual and the two theoretically maximum possible speeds, the data are grouped into successive observation windows (see last column of Table 1). Here, we chose to compute the Spearman correlation because we expect a roughly monotonic relation and do not want our findings to be heavily affected by outliers. Since these observation windows each only contain a couple thousand slabs, they capture the degree to which the casting speed constraints were taken into account during a given production period. The resulting time series of correlations offers insights into the emergence of distinct production regimes as well as the planning paradigms underlying these regimes. Then, we try to confirm the outcome of the correlation analysis by inspecting another measurement series that quantifies the steel mass flow generated by the continuous casting machine. As its name suggests, this time series is closely related to the mass flow constraint, while it directly reflects the economic output of the factory.

Step 3 Downgrading models and comparison of regime performance

Next, we will contrast the mass flow output of the individual production regimes assuming that slabs might start to break as soon as the mass flow constraint is violated. To this end, any measurement outliers that exceed a certain mass flow threshold are downsized according to two different probability models (i.e., binary and linear) before

Table 1 Sample screenshot of production data

\begin{tabular}{lllllll}
\hline Sequence ID & Heat ID & Width/mm & Thickness/mm & Weight/t & Duration/s & Observation window \\
\hline 1 & 1.1 & 1800 & 55 & 25 & 450 & 1 \\
1 & 1.2 & 1750 & 65 & 35 & 635 & 1 \\
$\ldots$ & $\ldots$ & $\ldots$ & $\ldots$ & $\ldots$ & $\ldots$ & $\ldots$ \\
$N$ & $N .1$ & 1800 & 65 & 30 & 545 & $M$ \\
$N$ & $N .2$ & 1750 & 70 & 32 & 580 & $M$ \\
\hline
\end{tabular}


the mean mass flow for each regime is estimated. In particular, we are interested in the threshold value (the applied mass flow threshold is varied between 0.055 and $0.075 \mathrm{t} / \mathrm{s}$ ) at which the regimes exhibit the same mean output. Technically, this value should reflect the theoretically maximum possible mass flow provided that the regime transitions happened under the premise to keep the overall performance of the continuous caster unchanged.

In the case of the binary probability model, all peaks above the threshold are reduced to zero (see Fig. 2), whereas the second model lowers the peaks with a probability that linearly depends on the amount by which the threshold is surpassed.

Additionally, we study how the performance of the linear model is influenced by a model-inherent factor $a$, and we alter this linear factor up to the point where the binary model is retrieved. The factor $a$ basically determines the relevance of the mass flow constraint. A larger value implies a better tolerance to constraint violations. For each regime, the mass flow associated with the binary model $M_{\text {bin }}^{n}$ can be described by

$M_{\text {bin }}^{n}\left(x_{n}\right)=\left\{\begin{array}{l}x_{n}, \text { if } \quad x_{n} \leq t \\ 0, \text { if } \quad x_{n}>t\end{array}\right.$,

where $n$ is the regime number; $x_{n}$ is the input mass flow of the $n$th regime; and $t$ is the mass flow threshold. The linear model leads to the mass flow $M_{\text {lin }}^{n}$,

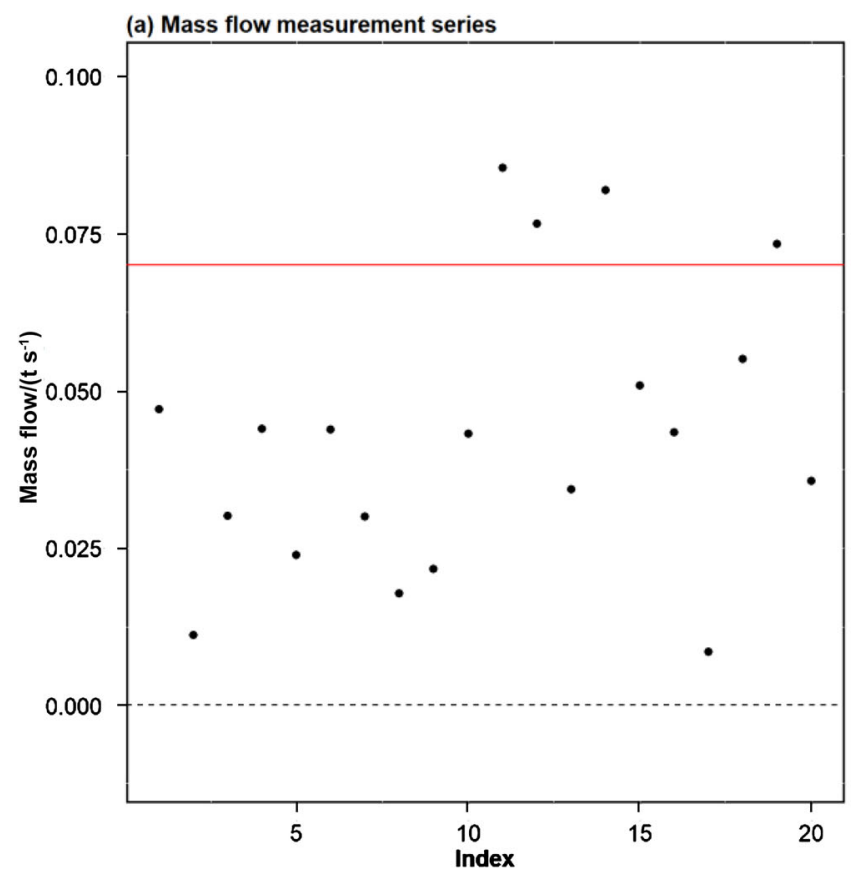

$M_{\operatorname{lin}}^{n}(x)=\left\{\begin{array}{ccll}x_{n}, \text { if } & x_{n} \leq t & \\ x_{n}, \text { if } & x_{n}>t \quad \text { and } & p>\min \left(\frac{x_{n}-t}{a}, 1\right) \\ 0, \text { if } & x_{n}>t & \text { and } & p \leq \min \left(\frac{x_{n}-t}{a}, 1\right)\end{array}\right.$

where $p$ denotes a $U(0,1)$-distributed random number. The second and third cases in Eq. (4) both depict situations where the threshold of the system is violated, and subsequently, the production process carries on as usual $\left(p>\min \left(\frac{x_{n}-t}{a}, 1\right)\right)$ or stops completely $\left(p \leq \min \left(\frac{x_{n}-t}{a}, 1\right)\right)$. For very small $a$, both models are equivalent:

$\lim _{a \rightarrow 0}\left(M_{\text {lin }}^{n}\right)=M_{\text {bin }}^{n}$.

Finally, our procedure concludes in an intuitive representation of the mean mass flow values belonging to the individual production regimes. This allows us to interpret the impact of the linear factor and the mass flow threshold on the regime performance. On top of that, we are granted a general understanding of the theoretically maximum mass flow beyond which serious quality problems occur and the economic output drops.

\section{Results and discussion}

This chapter demonstrates the results obtained from the procedural steps listed in Sect. 3.

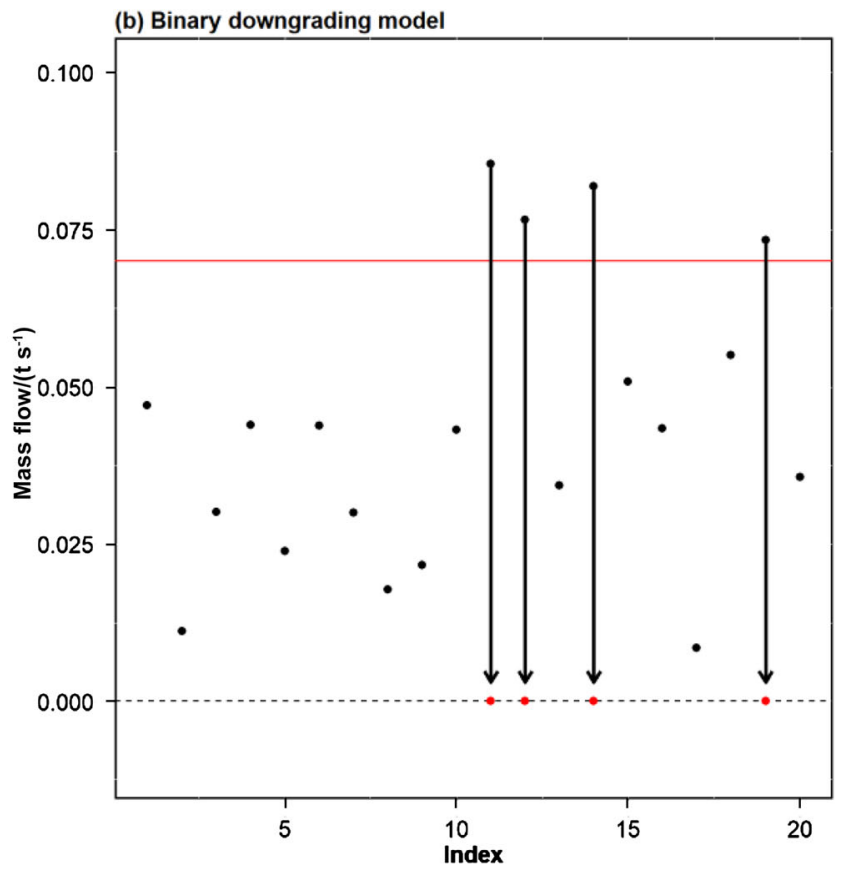

Fig. 2 Explanation of binary downgrading model. a Actual measurement series of $x_{n} ; \mathbf{b}$ all peaks above mass flow threshold (red line) downsized to zero (dashed line) 
Step 1 Computation of casting speeds and comparison with randomized casting speeds

After having calculated the various casting speeds (i.e., actual and theoretical), we show the actual speed for a period of 1000 slabs selected at random, which corresponds to a production duration of roughly 5 days (see Fig. 3). Apparently, the casting speed may be constant over short periods (10-100 slabs), but also typically fluctuates a lot inside a corridor of approximately $2 \mathrm{~m} / \mathrm{min}$. Our finding is in line with the previously established notion that casting speeds ideally should not vary much. However, in practice, casting machine operators might not be able to universally adhere to this rule of thumb.

This is further underlined by Fig. 4, as it displays the mean casting speed and its standard deviation for a randomly selected period of 50 production campaigns (5000-15,000 slabs). While the speed standard deviations-represented by the error bars - can be very minuscule, they tend to grow as big as $0.5 \mathrm{~m} / \mathrm{min}$ at times. Nevertheless, it seems that the casting machine operators attempt to minimize speed fluctuations since thoroughly shuffling the identification labels of the production campaigns and keeping the actual speed values fixed would yield a much higher average of the intra-sequence standard deviation (approximately $0.5 \mathrm{~m} / \mathrm{min}$ ) than that suggested by the industrial data $(0.27 \mathrm{~m} / \mathrm{min})$ (see Supplementary Material: Fig. S1).

Step 2 Correlation analysis and identification of production regimes

Next, we divide the entire database into successive observation windows - each containing a couple of thousand slabs. Now, it is possible to compare the actual casting speed to both theoretical speeds introduced earlier. Figure 5 depicts the development of the Spearman correlation between these casting speeds. The correlations are measured for consecutive observation windows that comprise several 1000 slabs, whereby the orange (blue) line corresponds to machine length (mass flow) constraint. Analogous plots for different window sizes are enclosed in the supplements (see Supplementary Material: Fig. S2). From the significant increment in the blue graph between the 17 th and the 20th windows, it becomes evident that the mass flow constraint was taken into account much more rigorously in the second half of the production history. Thus, $w$ impacts the casting speed in practice, because it is an integral part of the casting speed formula entailed by the mass flow constraint (i.e., $\left.v_{\text {mass }}=m /(d w \theta \rho)\right)$. On the other hand, the orange graph belonging to the machine length constraint (i.e., $v_{\text {solid }}=k \times l_{\max } / \theta^{2}$ ) experiences a downswing around the 20th window but recovers afterward. In light of this recovery, we assume that $\theta$ still remains the key variable with respect to the casting speed since it is included in both casting speed formulae. However, it comes as a bit of a surprise that, at the end, the mass flow is somewhat regarded to a higher degree than the machine length constraint: technically, the machine length constraint is a hard constraint (i.e., every individual slab needs to abide by it), while the mass flow constraint can be ignored occasionally as long as the tundish does not run out of steel in the long term. Maybe, the operators prefer a single casting speed formula that includes both the width and the thickness over using two separate formulae.

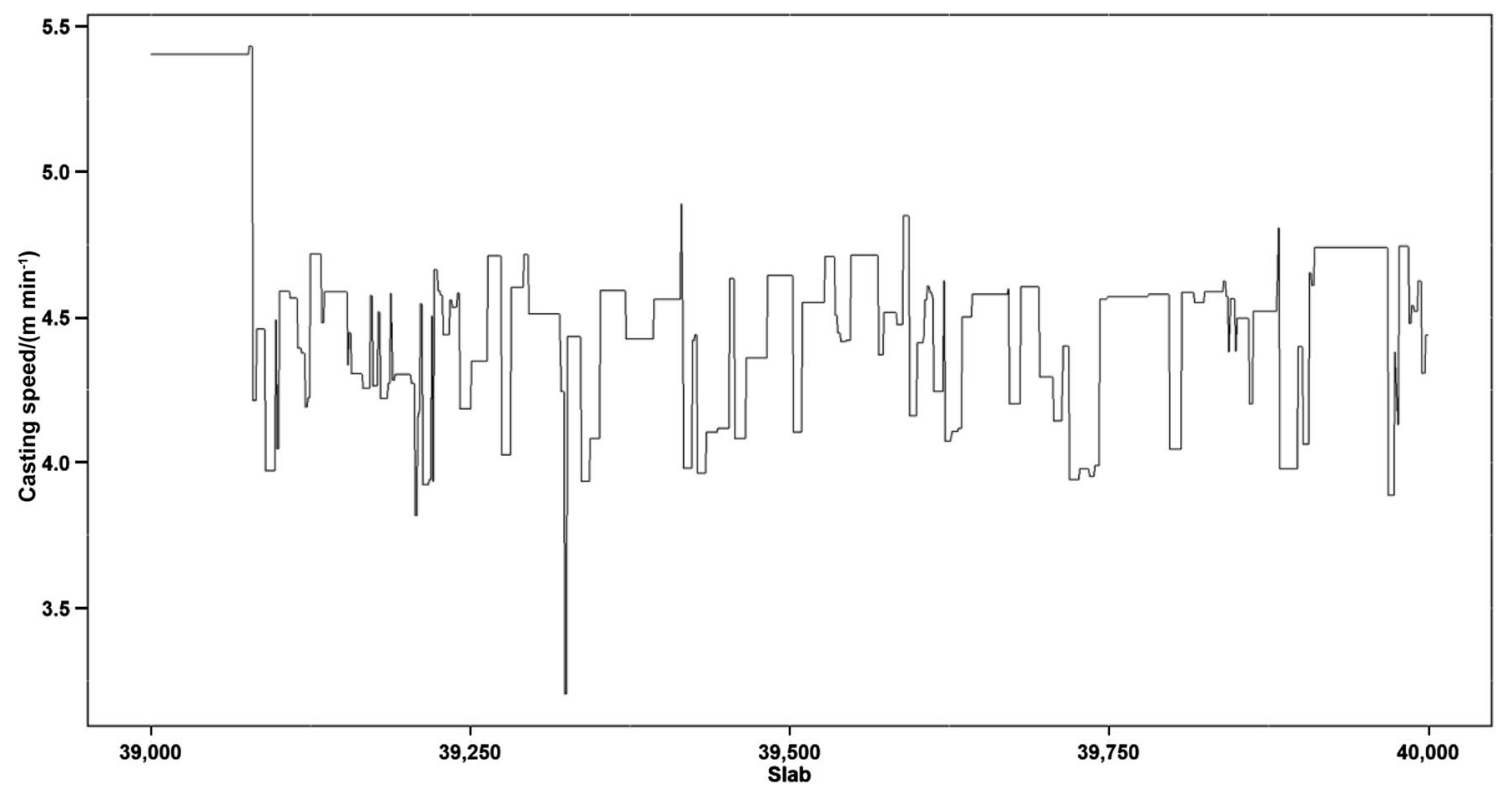

Fig. 3 Time series plot showing evolution of actual casting speed for a randomly selected period of 1000 slabs 


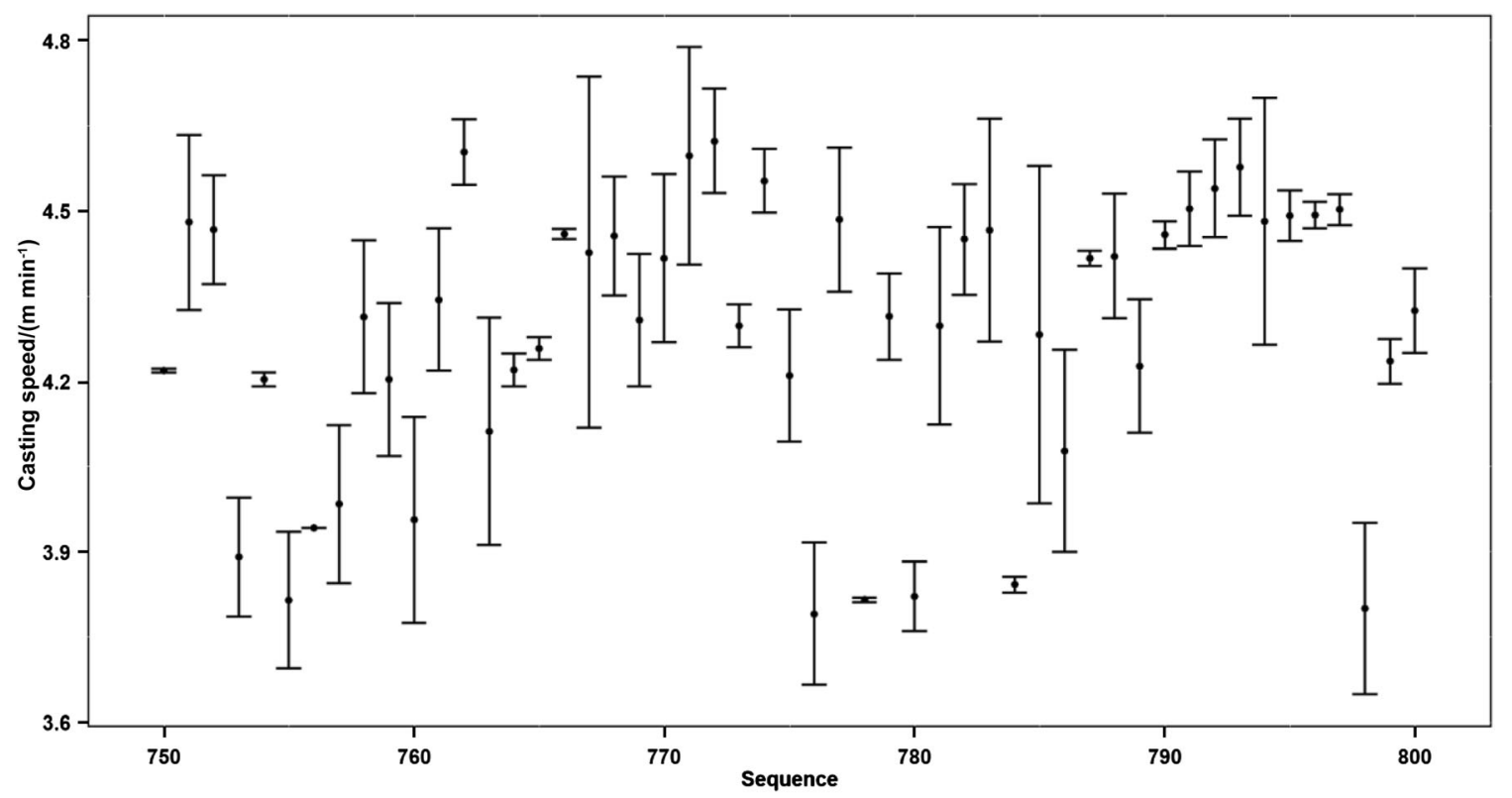

Fig. 4 Mean casting speeds as a function of sequence ID for a randomly selected period of 50 production campaigns

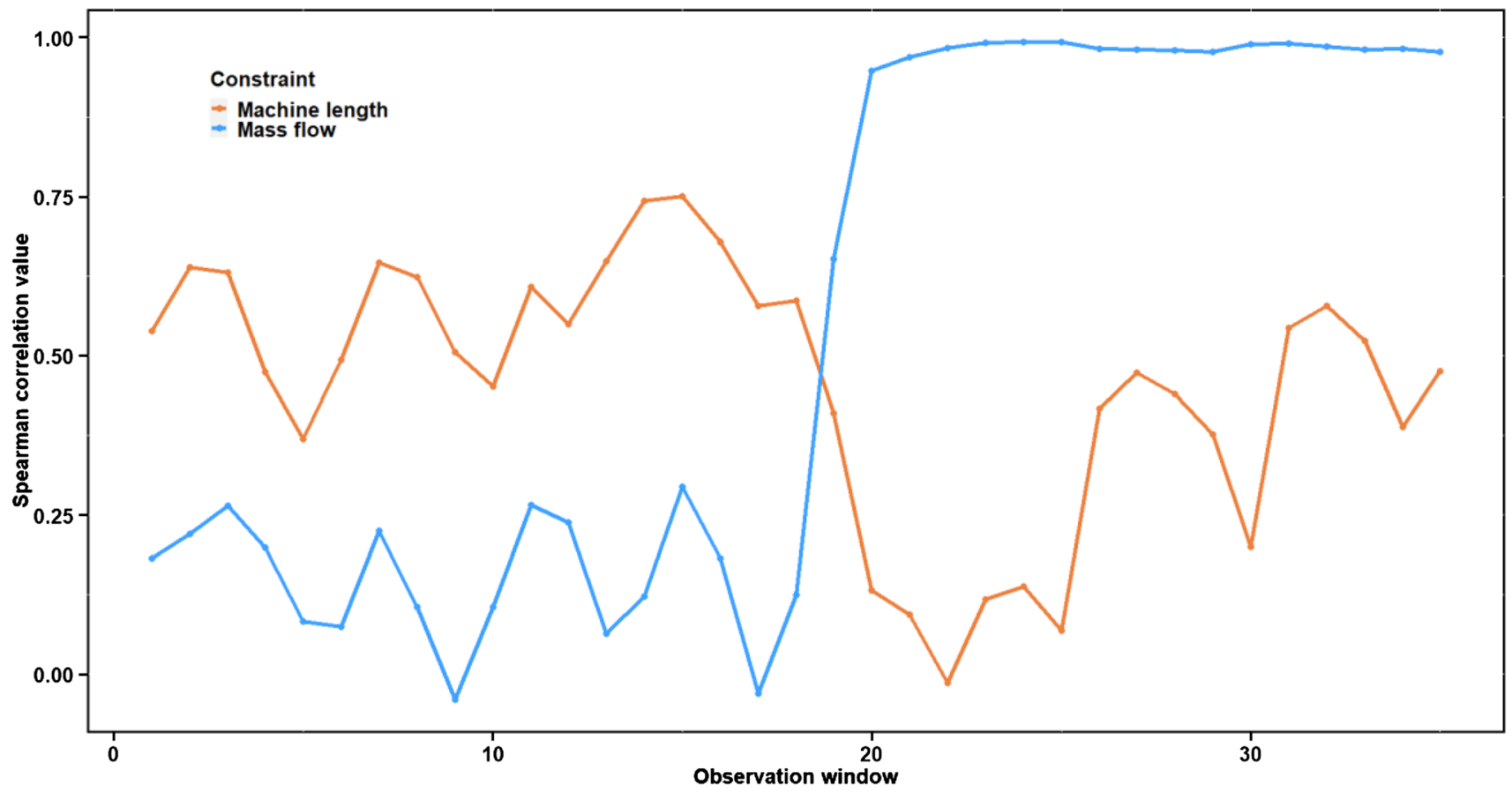

Fig. 5 Evolution of Spearman correlation between actual casting speed and two theoretically maximum possible casting speeds associated with machine length constraint and mass flow constraint, respectively

Figure 6 documents the mean steel mass flow created by $\mathrm{CC}$ machine for all production campaigns and, interestingly, it mirrors the regime shift concerning the mass flow constraint. Therefore, on the basis of the similarities between Figs. 5 and 6 as well as the sharp cut-off in Fig. 6, we deduce that the increased prominence of the mass flow constraint was achieved through the restriction of said mass flow at $0.06 \mathrm{t} / \mathrm{s}$. Note that the regime changes in Figs. 5 and
6 do not perfectly align due to variations of sequence length (100-300 slabs).

Step 3 Downgrading models and comparison of regime performance

As we already know, the main reason for a restriction of the mass flow lies in the presumed breaking of the steel slab that is affiliated with a continuing violation of the mass 


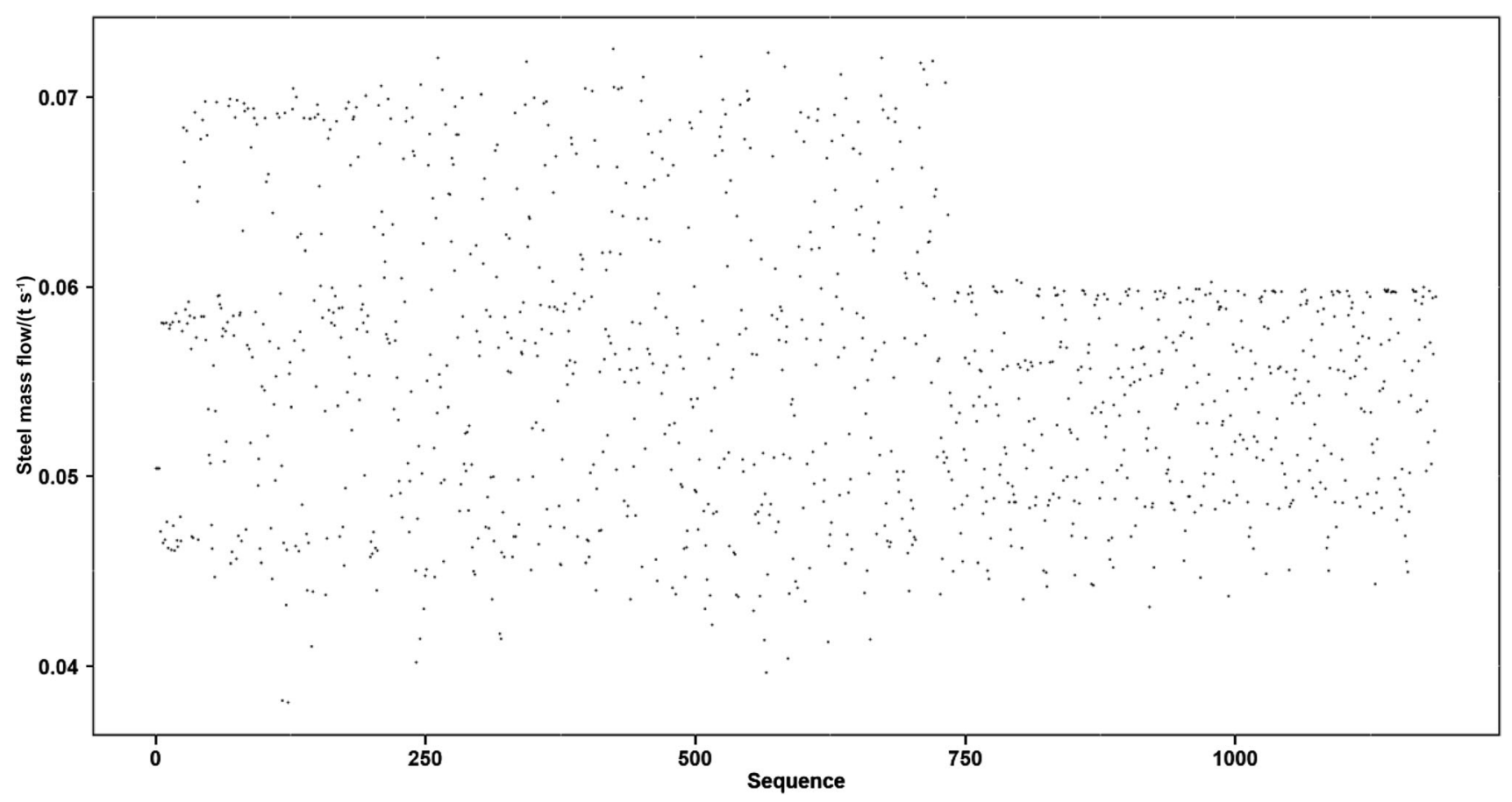

Fig. 6 Mean mass flow as a function of sequence ID for all production campaigns

flow constraint. Henceforth, we want to elaborate on the trade-off decision stated in Sect. 1, where an augmented production output due to higher casting speeds competes with an elevated risk of quality issues. Figure 7 contrasts the average mass flow ( $y$-axis) for both previously detected production regimes provided that the output of any production campaign behaves in a binary fashion and declines to zero as soon as the measured mass flow exceeds a certain threshold ( $x$-axis). The mean mass flow is measured as a function of the mass flow threshold beyond which mass flow is definitely reduced to zero, while the red (green) line corresponds to the early (late) regime.

Clearly, for mass flow thresholds greater than $0.07 \mathrm{t} / \mathrm{s}$, the preceding regime outperforms the later regime (ergo: the one associated with a more stringent application of the mass flow constraint). Thus, if the binary probability model accurately reflected the reality, one could assume that the limit of $0.06 \mathrm{t} / \mathrm{s}$ was implemented to have some sort of safety buffer toward the definite physical boundary beyond which the early strategic regime turns out to be superior. Unfortunately, we cannot be sure whether the slab starts to break immediately when the mass flow threshold is surpassed or whether there is some room for minor negligence regarding this constraint.

To this end, we designed a linear probability model, where the downsizing probability depends on the extent of the constraint violation, and we added a factor that controls the downsizing (see Sect. 3). Accordingly, the output difference between our two regimes as a function of the mass flow threshold and the linear factor is featured in the visual output representation mentioned in the beginning (see Fig. 8). In Fig. 8, the mean mass flow is measured as a function of the mass flow threshold as well as a linear factor that controls the outlier downsizing, while the green (red) area corresponds to those values of the input variables for which the late (early) regime is superior. As anticipated, we recovered the binary model by decreasing the linear factor to zero. More importantly, given the imposed cut-off mass flow of $0.06 \mathrm{t} / \mathrm{s}$ (see Fig. 6), the linear factor, for which the mean output values for both regimes match each other, looks to be in the vicinity of 0.1 . We believe that the value of 0.1 refers to potential situations where the machine length constraint permits a higher casting speed than the mass flow constraint (e.g., extremely thin but wide slabs) and, for very short periods, it is conceivable to exceed the rate at which liquid steel is supplied to the tundish.

Thus, subject to the condition that the machine operators wanted to enforce the reduction in the mass flow without affecting the overall production output of the continuous caster, it is fair to say that from their point of view, slightly overshooting the imposed cut-off mass flow will not necessarily lead to immediate quality problems. As long as the machine length constraint is fulfilled, the operators probably do not consider the mass flow balance to be a hard constraint that has to be complied at any cost. Instead, meticulously regulating the mass flow is perceived as a desirable objective that should be tackled once the build-up 


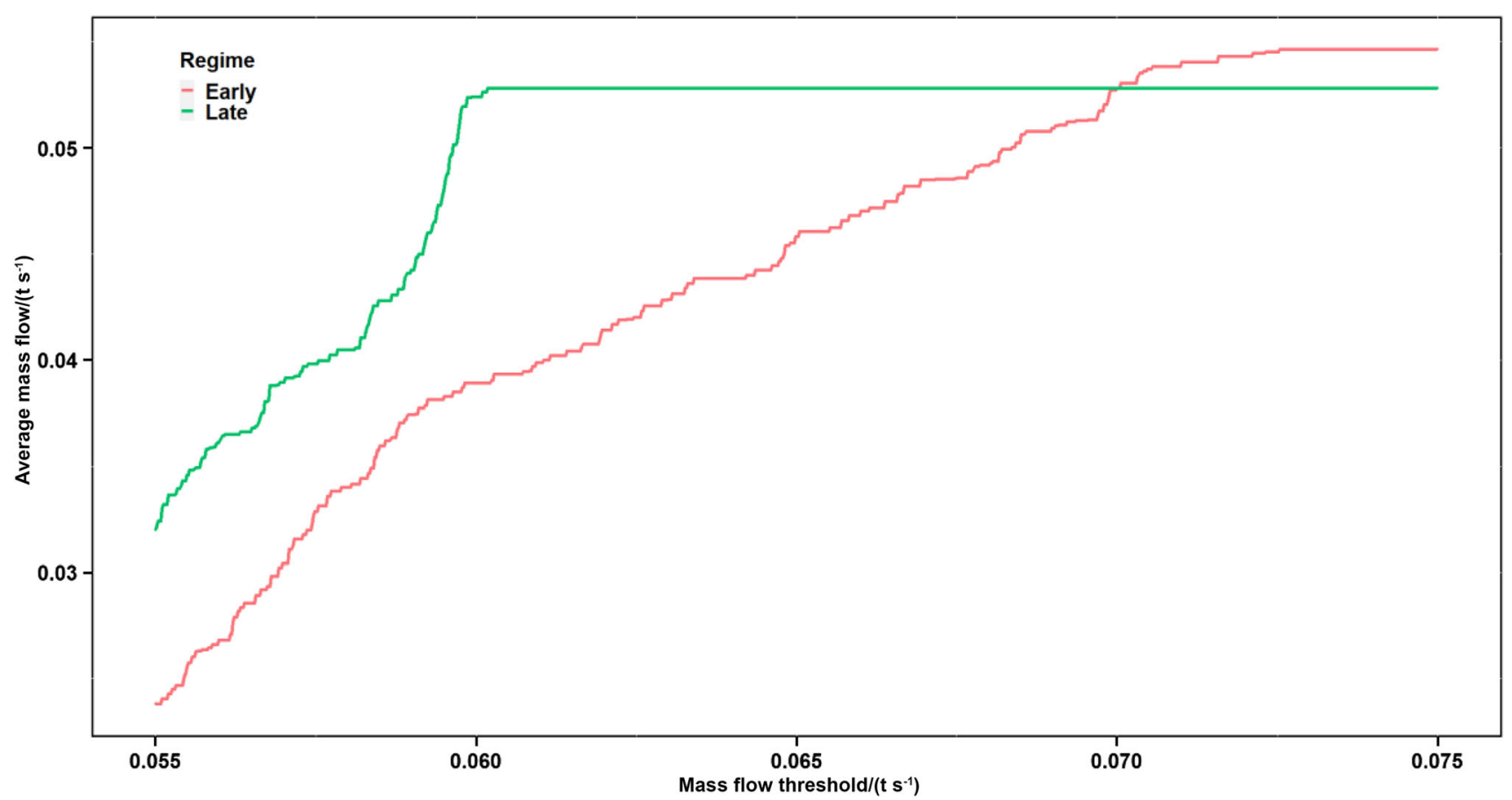

Fig. 7 Mean mass flow for two distinct production regimes (see Fig. 6) that differ in their degree to which they respect mass flow constraint

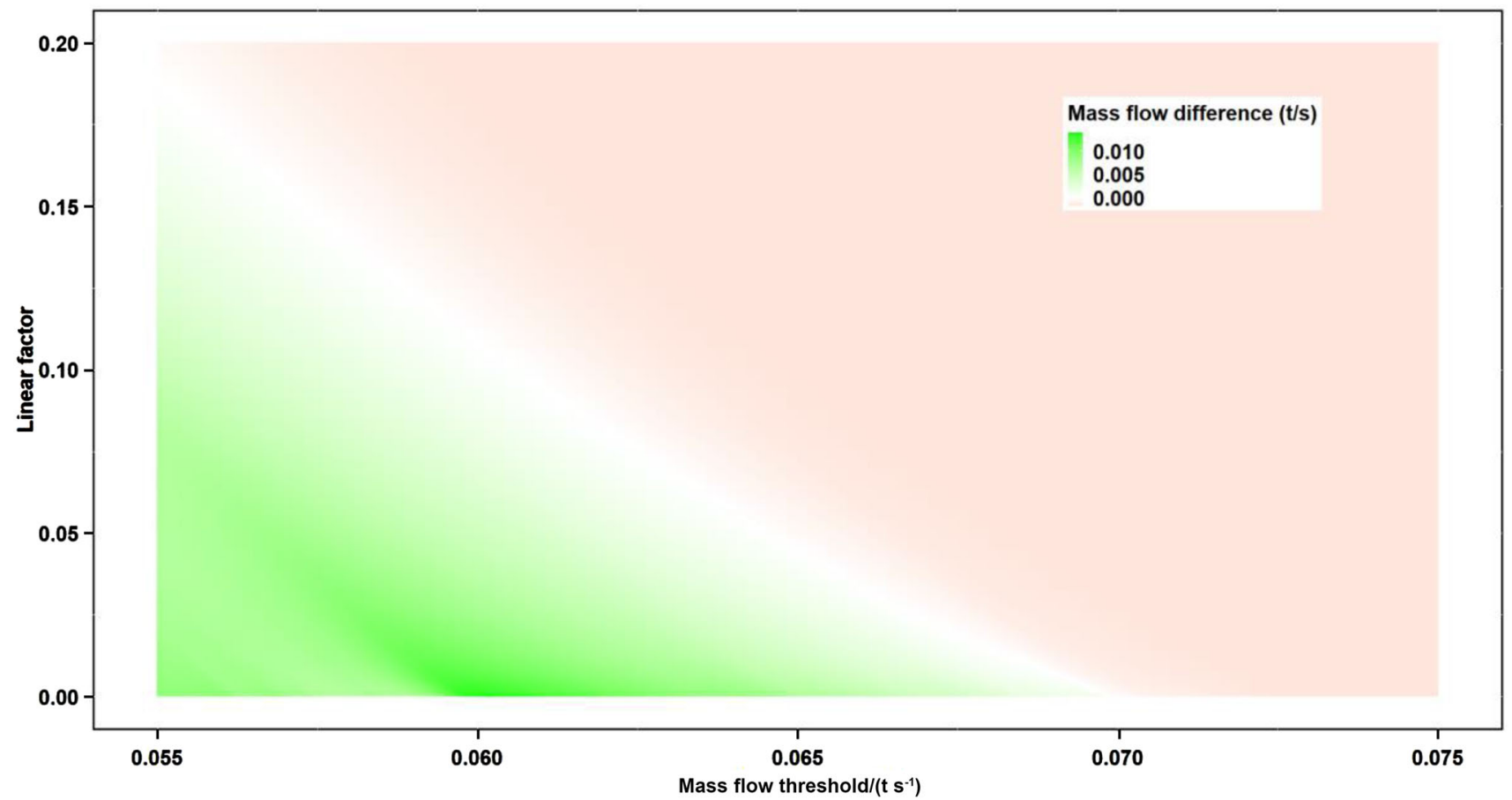

Fig. 8 Mean mass flow difference between two distinct production regimes (see Fig. 6) that differ in their degree to which they respect mass flow constraint

production phase following the factory commissioning (i.e., the first production regime) is completed.

\section{Conclusions}

In summary, we have offered a novel perspective on casting speed fluctuations by investigating the effect of the slab width on the appropriate casting speed with the help of 
industrial data. This effect was traced back to the mass flow constraint (i.e., $v_{\text {mass }}=m /(d w \theta \rho)$ ) which governs the balance between steel input toward the tundish and steel output away from the tundish. As opposed to the machine length constraint (i.e., $v_{\text {solid }}=k \times l_{\max } / \theta^{2}$ ), the mass flow constraint does not only bear in mind $\theta$, but also $w$.

We have found that in the steel plant of interest, a strategic adjustment of the maximally measured steel mass flow has taken place. Most likely, the plant's decision to pay more attention to the mass flow constraint was made due to economic reasons since higher mass flows entail larger profits only if the product quality is acceptable. By comparing the mean mass flow before and after the strategy change-provided that violations of the mass flow constraint diminish the casting quality - we shed light on the economic implications of this operational decision. The resulting visualization technique takes into account the height of the mass flow threshold above which serious quality issues occur as well as the possibility that downgrading might happen probabilistically. We conclude that subsequent to a steel plant's build-up production phase, the mass flow constraint plays an important role in practice, but it can be ignored occasionally if the machine length constraint is respected at the same time.

Supplementary Information The online version contains supplementary material available at https://doi.org/10.1007/s42243021-00729-5.

Funding Open Access funding enabled and organized by Projekt DEAL.

Open Access This article is licensed under a Creative Commons Attribution 4.0 International License, which permits use, sharing, adaptation, distribution and reproduction in any medium or format, as long as you give appropriate credit to the original author(s) and the source, provide a link to the Creative Commons licence, and indicate if changes were made. The images or other third party material in this article are included in the article's Creative Commons licence, unless indicated otherwise in a credit line to the material. If material is not included in the article's Creative Commons licence and your intended use is not permitted by statutory regulation or exceeds the permitted use, you will need to obtain permission directly from the copyright holder. To view a copy of this licence, visit http://creativecommons. org/licenses/by/4.0/.

\section{References}

[1] B.G. Thomas, Continuous Casting, in: K.H. J. Buschow, R. Cahn, M. Flemings, B. Ilschner, E.J. Kramer, S. Mahajan, D. Apelian (Eds.), The Encyclopedia of Materials: Science and Technology, Elsevier, Oxford, UK, 2001.

[2] J. Tu, G.H. Yeoh, C. Liu, Computational fluid dynamics: a practical approach, 3rd ed., Butterworth-Heinemann, Elsevier Ltd., 2019.

[3] V. Smil, Still the iron age: iron and steel in the modern world, Butterworth-Heinemann, Elsevier Inc., 2016.

[4] B.G. Thomas, Steel Res. Int. 89 (2018) 1700312.

[5] C. Li, B.G. Thomas, in: 85th Steelmaking Conference Proceedings, ISS, Warrendale, PA, USA, 2002, pp. 109-130.

[6] Y. Wang, L. Zhang, ISIJ Int. 50 (2010) 1783-1791.

[7] Q.Y. Zhang, X.H. Wang, J. Iron Steel Res. Int. 17 (2010) No. 8, 15-19.

[8] A. Özgür, Y. Uygun, M.T. Hütt, Comput. Ind. Eng. 151 (2021) 106606.

[9] D. Merten, M.T. Hütt, Y. Uygun, A network analysis of decision strategies of human experts in steel production. https://arxiv.org/ abs/2112.01991.

[10] C. Li, B.G. Thomas, in: The Brimacombe Memorial Symposium, Met Soc., CIM, Vancouver, Canda, 2000, pp. 595-611.

[11] B.G. Thomas, A. Denissov, H. Bai, in: ISS 80th Steelmaking Conference Proceedings, ISS, Chicago, IL, USA, 1997, pp. 375-384.

[12] Y.A. Meng, B.G. Thomas, Metall. Mater. Trans. B 34 (2003) 685-705.

[13] H.J. Shin, B.G. Thomas, G.G. Lee, J.M. Park, C.H. Lee, S.H. Kim, in: MS\&T 2004 Conference Proceedings, AIST, Warrendale, PA, USA, 2004, pp. 11-26.

[14] J.K. Brimacombe, Can. Metall. Quart. 15 (1976) 163-175.

[15] K. Schwerdtfeger, ISIJ Int. 38 (1998) 852-861.

[16] J.E. Camporredondo S., F.A. Acosta G., A.H. Castillejos E., E.P. Gutiérrez M., R. González de la P., Metall. Mater. Trans. B 35 (2004) 561-573.

[17] B.G. Thomas, Metall. Mater. Trans. B 33 (2002) 795-812.

[18] K. Miłkowska-Piszczek, M. Dziarmagowski, A. Buczek, J. Pióro, Arch. Mater. Sci. Eng. 57 (2012) 75-79.

[19] Y. Liu, X.D. Wang, M. Yao, X.B. Zhang, H. Ma, Z. Wang, J.C. Ma, X. Wang, G.Q. Shi, Ironmak. Steelmak. 41 (2014) 748-755.

[20] L.P. Li, X.H. Wang, X.X. Deng, C.X. Ji, J. Iron Steel Res. Int. 22 (2015) 1-9.

[21] G.G. Lee, H.J. Shin, S.H. Kim, S.K. Kim, W.Y. Choi, B.G. Thomas, Ironmak. Steelmak. 36 (2009) 39-49.

[22] S.Z. Wu, J.M. Zhang, Z.Z. Li, J. Iron Steel Res. Int. 17 (2010) No. 8, 6-9.

[23] R.B. Mahapatra, J.K. Brimacombe, I.V. Samarasekera, Metall. Mater. Trans. B 22 (1991) 875-888.

[24] J.X. Fu, W.S. Hwang, J.S. Li, S.F. Yang, Z. Hui, Steel Res. Int. 82 (2011) 1266-1272.

[25] P.H. Dauby, Metall. Res. Technol. 109 (2012) 113-136.

[26] X. Deng, C. Ji, Y. Cui, L. Li, X. Yin, Y. Yang, A. McLean, Ironmak. Steelmak. 44 (2017) 461-471. 